\title{
DÜBLIN
}

Technological University Dublin

ARROW@TU Dublin

2004-01-01

\section{Control loop performance assessment: a classification of methods}

\author{
Niall O'Connor \\ Technological University Dublin \\ Aidan O'Dwyer \\ Technological University Dublin, aidan.odwyer@tudublin.ie
}

Follow this and additional works at: https://arrow.tudublin.ie/engscheleart

Part of the Controls and Control Theory Commons

\section{Recommended Citation}

O'Connor, Niall and O'Dwyer, Aidan : Control loop performance assessment: a classification of methods. Proceedings of the Irish Signals and Systems Conference, pp. 530-535, Queens University Belfast, July, 2004 doi:10.21427/m36r-z414

This Conference Paper is brought to you for free and open access by the School of Electrical and Electronic Engineering at ARROW@TU Dublin. It has been accepted for inclusion in Conference papers by an authorized administrator of ARROW@TU Dublin. For more information, please contact arrow.admin@tudublin.ie, aisling.coyne@tudublin.ie,gerard.connolly@tudublin.ie.

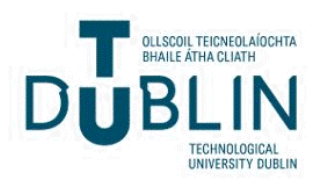




\title{
Control Loop Performance Assessment: A Classification of Methods
}

\author{
Niall O'Connor ${ }^{\phi}$ and Aidan O'Dwyer* \\ ${ }^{\phi}$ School of Control Systems and Electrical \\ Engineering, \\ Dublin Institute of Technology \\ Kevin St., Dublin 8, IRELAND \\ E-mail: ${ }^{\phi}$ alf_fonzo@hotmail.com \\ * School of Control Systems and Electrical \\ Engineering, \\ Dublin Institute of Technology \\ Kevin St., Dublin 8, IRELAND \\ E-mail:*aidan.odwyer@dit.ie
}

\begin{abstract}
This paper presents an overview of a number of controller performance assessment techniques. The techniques discussed are divided into five categories, namely, time domain assessment, frequency domain assessment, minimum variance control (MVC) as a benchmark, statistical analysis, and other more 'problem specific' assessment techniques. Recent work, by various authors, in each of the five categories is outlined.
\end{abstract}

\section{INTRODUCTION}

According to [1] monitoring of process variables is useful, not only for assessing the status of the process, but also for controlling product quality. According to [2], in the testing of thousands of control loops in hundreds of operating plants, Techmation Inc. and others have found that more than $30 \%$ of the automatic control loops actually increase variability over manual control due to poor controller tuning. One reason why so many control loops perform poorly is that there is often numerous (more than a thousand) loops in a large process plant and not enough control engineers to maintain every loop.

In [3] Jamsa-Jounela et al. make the point that in order to ensure highest product quality it is essential to maintain the control system in an adequate manner. In [4] Vishnubhotla et al. discuss how the current standard practice for industrial process control is to install DCS (Distributed Control Systems) and PLC control system platforms. These system platforms accumulate large volumes of process data, but there are very few data mining tools.

It should be obvious, therefore, that there is a strong need for automatic assessment and monitoring of control loop performance. The goal of monitoring should be to provide information that can be used to assess the current status of the existing controller and to assist control engineers in deciding whether redesign is necessary [5]. When the controller performance is determined to be inadequate, it is important to ascertain whether an acceptable level of performance can be achieved with the existing control structure [6].

With these goals in mind, the next step is to review some of the existing loop performance assessment techniques. It was decided to divide the assessment techniques into the following categories:
1. Time domain assessment,

2. Frequency domain assessment,

3. Minimum variance control (MVC) as a benchmark,

4. Statistical analysis techniques, and

5. Other more 'problem specific' assessment techniques

\section{TIME DOMAIN ASSESSMENT}

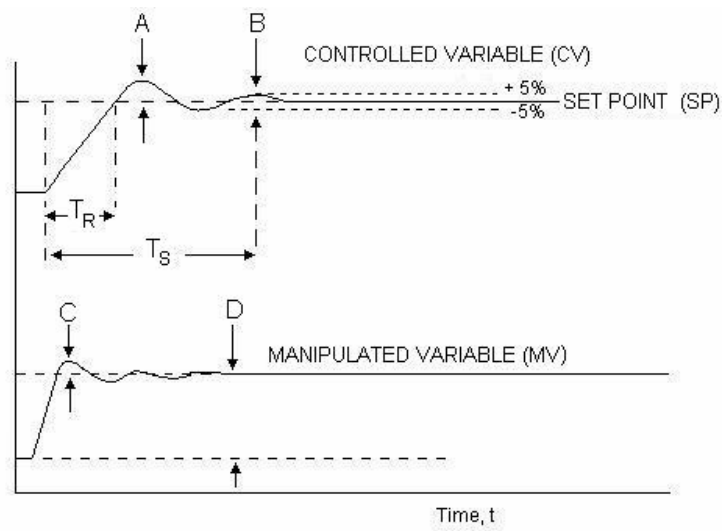

Figure (1). Typical transient response of a feedback control system to a step set point change

The dynamic response characteristics of a system may be accurately assessed using a number of useful time domain measures. These measures include rise time, settling time and integral error measures, see Figure (1). The rise time $\left(T_{r}\right)$ is defined as the time from the step change in the set point until the controlled variable first reaches the new set point [7]. A short rise time is usually desired. The settling time $\left(\mathrm{T}_{\mathrm{s}}\right)$ is defined as the time the system takes to attain a 'nearly constant' value, usually + or -5 percent of its final value [7]. This measure is related to the rise time 
and decay ratio. A short settling time is usually desired.

The integral error measures indicate the cumulative deviation of the controlled variable from its set point during the transient response. The Integral of Absolute Error (IAE) criterion is determined from the sum of areas above and below the setpoint. It is an appropriate measure of control performance when the effect on control performance is linear with the deviation magnitude. The Integral of Squared Error (ISE) criterion is appropriate when large deviations cause greater performance degradation than small deviations. The Integral of Time multiplied by Absolute Error (ITAE) criterion penalizes deviations that endure for a long time. The Integrated Error (IE) criterion is not normally used because positive and negative errors cancel in the integral, resulting in the possibility of large positive and negative errors giving a small IE [7]. The formulae for calculating the integral error measures are given below:

$$
\begin{aligned}
& I A E=\int_{0}^{\infty}|S P(t)-C V(t)| \cdot d t \\
& I S E=\int_{0}^{\infty}[S P(t)-C V(t)]^{2} \cdot d t \\
& I T A E=\int_{0}^{\infty} t \cdot|S P(t)-C V(t)| \cdot d t \\
& I E \quad=\int_{0}^{\infty}[S P(t)-C V(t)] \cdot d t
\end{aligned}
$$

In [3] Jamasa-Jounela et al. present a set of performance indices appropriate to process monitoring and assessment. These indices include IAE, ITAE, rise time and settling time. In [8] Swanda and Seborg have developed a new methodology to assess the performance of PI controllers from closed loop response data for a setpoint step change. This method is based on two new dimensionless performance indices, the dimensionless settling time and the dimensionless IAE. This methodology is also applicable to PID controllers. In [9] Horch and Stattin extend this method to analyse the settling timenormalised by the apparent process time delay-of a setpoint step response. In [10] Ruel discusses a number of metrics used to assess loop performance. These include IAE, setpoint crossing, and average error. In [11] Huang and Jeng assess a simple feedback system by analysing IAE and rise time observed from the response of the system to a step setpoint change. Optimal IAE's and associated rise times are computed. Comparing its current IAE to the optimal IAE allows an assessment of the performance of the system.

Explained in more detail below, there are a variety of other time domain measures that may be used to assess a systems performance. These include offset, decay ratio, manipulated variable overshoot, maximum deviation of the controlled variable, and magnitude of the controlled variable in response to a sine disturbance. Offset is defined as the difference between the final, steady state value of the set point and of the controlled variable. In most cases, a zero steady state offset is desired [7]. The decay ratio
(B/A), see Figure (1), is the ratio of neighbouring peaks in an underdamped controlled-variable response. Usually, periodic behaviour with large amplitudes is avoided in process variables; therefore, a small decay ratio is usually desired, and an overdamped response is sometimes desired [7]. The manipulated variable overshoot (C/D), see Figure (1), is of concern because the manipulated variable is also a process variable that influences performance. Some large variations can cause long-term degradation in equipment performance. The overshoot is the maximum amount that the manipulated variable exceeds its final steady state value and is usually expressed as a percentage of the change in manipulated variable from its initial to its final value. Some overshoot is acceptable in some cases [7]. The maximum deviation of the controlled variable from the set point is an important measure of the process degradation experienced due to disturbances. Usually a small value is desirable so that the process variable remains close to its set point [7]. In many cases the disturbance is composed predominantly of one or a few sine waves. Therefore, the behaviour of the control system in response to sine inputs is of great practical importance, because through this analysis the relationship between the frequency of the disturbances and the control performance is deduced. Control performance is assessed by measuring the amplitude of the output sine wave; the metric is often expressed as the ratio of the output to input sine wave amplitudes [7].

In [12] Stanfelji et al. present a method for monitoring and diagnosing the performance of single loop-control systems based primarily on normal operating data. This method involves analysing the autocorrelation and cross correlations of a time series of control loop variables. In [13] Hagglund describes a procedure for the automatic detection of sluggish control loops obtained from conservatively tuned controllers. The 'idle index' describes the relation between the times of positive and negative correlation between the control and measurement signal increments. From this index the sluggishness of the control loop can be determined.

\section{FREQUENCY DOMAIN ASSESSMENT}

According to [14] traditional measures such as overshoot, rise time, decay ratio, settling time and the ISE are difficult to translate into an economic measure so as to justify process or control system redesign. They state, however, that frequency domain measures can be used to provide a measure of performance that can be translated into an economic measure. This section will review some of the more common frequency domain assessment methods. Three different types of plots are commonly used to graphically illustrate the frequency response of a controlled system, see Figure (2). These three plots are the Nyquist, Bode and Nichols plots. Nyquist plots, also called polar plots, may be obtained by either plotting the real versus the imaginary part of the frequency domain transfer function, $G(j w)$ (using rectangular coordinates), or by plotting the magnitude at a particular phase angle of $\mathrm{G}(\mathrm{jw}$ ) (using polar coordinates). Bode plots require two curves to be plotted; these plots show how the magnitude ratio and 
phase angle vary with frequency. The Nichols plot is a single curve in a coordinate system with phase angle as the abscissa and log modulus as the ordinate. Frequency is a parameter along the curve [15].
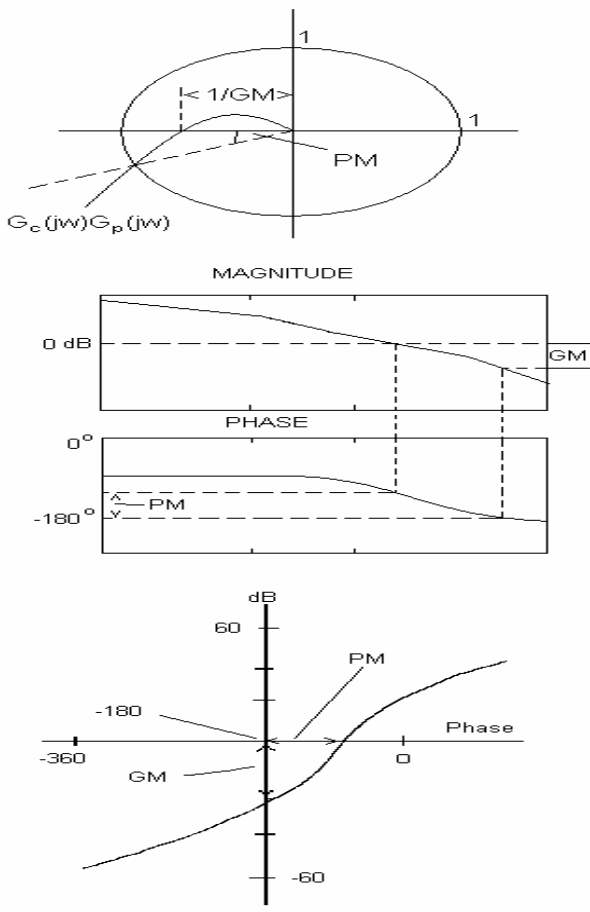

Figure (2) Nyquist, Bode and Nichols plots illustrating Gain and Phase margin.

Phase margin and gain margin are two commonly used assessment measures. Phase margin (PM) is defined as the angle between the negative real axis and a radial line drawn from the origin to the point where the open loop frequency domain transfer function intersects the unit circle. The bigger the phase margin the more stable the closed loop system. Phase margins of $45^{\circ}$ are often considered appropriate [15]. The gain margin (GM) is defined as the reciprocal of the intersection of the open loop frequency domain transfer function polar plot on the negative real axis. The bigger the gain margin, the more stable the system. Typically gain margin values of about 2 are recommended [15]. In [16] Astrom and Hagglund discuss a simple method for estimating the critical gain of a controlled system, from which the gain margin may be deduced.

The maximum closed loop log modulus, $\mathrm{L}_{\mathrm{cmax}}$, is another quantity used to assess performance in the frequency domain, see Figure (3). While the phase and gain margin specifications can sometimes give poor results when the shape of the frequency response curve is unusual, the maximum closed loop $\log$ modulus does not have this problem since it directly measures the closeness of the open loop frequency domain transfer function to the $(-1,0)$ point at all frequencies [15]. In [17] Chiou and $\mathrm{Yu}$ propose a monitoring procedure that identifies the maximum closed loop log modulus in two to three relay feedback experiments. In [5] Ju and Chiu present a monitoring procedure incorporating the FFT (Fast Fourier Transform) technique to identify $\mathrm{L}_{\mathrm{cmax}}$ on line. This proposed method addresses some of the problems in the method presented in [17] i.e. too many relay tests are required, the frequency search range is confined to the third quadrant, and the identified value of $\mathrm{L}_{\mathrm{cmax}}$ cannot be used on-line to redesign the controller. In [14] Belanger and Luyben propose a new test to locate the peak regulator log modulus. The test involves the insertion of a relay between the controlled variable and a given load disturbance model, with the feedback controller on automatic. This causes the plant to exhibit a sustained oscillation at the frequency where the $\mathrm{L}_{\text {cmax }}$ curve exhibits a peak. This test can be applied to both simulated models as well as existing plants.

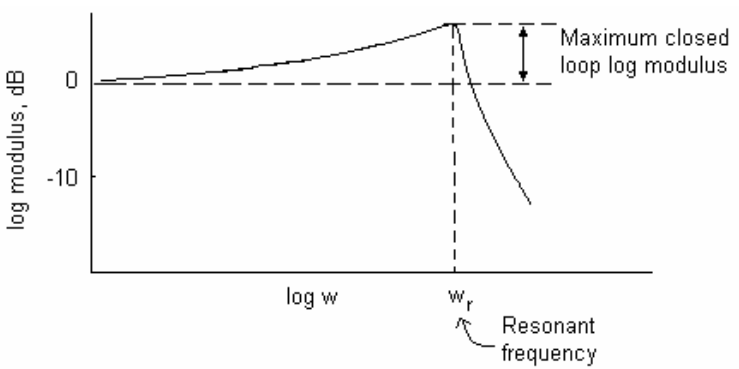

Figure (3). Plot illustrating the maximum closed loop $\log$ modulus $\mathrm{L}_{\mathrm{cmax}}$.

The capacity based method for quantifying controllability is a method used to quantitatively incorporate the economics of control into conventional steady-state design methods [15]. In [18] Elliot and Luyben outline a generic methodology called the capacity based economic approach that can be used to compare or screen preliminary plant designs by quantifying both steady-state economics and dynamic controllability. In [19] Elliot et al. demonstrate that the capacity based economic approach can be successfully applied to a large industrial scale process. In [20] Elliot and Luyben analyse the effectiveness of the capacity based economic approach when controlling a complex recycle system consisting of a reactor and two distillation columns.

In [21] Kendra and Cinar discuss a method used to estimate the closed loop transfer function of a system by exciting the reference input with a zero mean, pseudo random binary sequence and observing the process output and error response. Performance assessment is based on the comparison between the observed frequency response characteristics and the design specifications.

\section{Minimum Variance Control (MVC) AS A BENCHMARK}

According to [22] and [23], minimum variance control is considered the optimal feedback control provided that the process can be described by a linear transfer function with additive disturbance. In [24] Spring states that minimum variance is a better benchmark than zero variance for evaluating controller performance. Control systems cannot reduce the variance in product quality below the variance inherent in the process. On the basis of minimum variance, an investment in controller maintenance can be evaluated realistically. 
According to [4], this benchmark control may or may not be achievable in practice depending on process invertibilty and other process physical constraints. Also, it is worth noting that this technique requires knowledge of the process time delay, which may not always be available. However, as a benchmark, it provides useful information such as how much 'potential' there is to improve controller performance. In [25] Thornhill et al. make the point that minimum variance control may require excessively vigorous action of the manipulated variable and, as a result, can lead to maintenance problems for the actuators. This section presents a review of some of the papers available that discuss some of these details.

A number of papers are recommended that give an overview of the MVC method. In [23] Harris discusses how an estimate of the best possible control can be obtained by fitting a univariate time series to process data collected under routine control. In [26] Harris et al. discuss some of the concepts associated with assessing the effectiveness of a control system. Also discussed in this paper is how these concepts were initially developed using a performance benchmark of minimum variance control for SISO systems. In [25] Thornhill et al. examine some of the factors that influence the minimum variance performance measure of a SISO control loop. The authors show that, for an arbitrary controller, the calculated minimum variance benchmark is different for servo and regulator operation. In [27] Grimble discusses the use of the generalised minimum variance control law for control loop performance assessment and benchmarking. In [28] Huang and Shah discuss, in detail, some of the theory behind the MVC method.

Based on MVC theory, a performance index (the Harris index) was first introduced by Harris [23]. This index compares the actual variance in the process variable to that of a minimum variance controller. In [22] and [29] Desborough and Harris present a normalised performance index used to characterise the performance of control systems. This index provides a measure of the proximity of control to minimum variance control. Time domain and spectral interpretations of the index are discussed and a fast, simple on-line method for estimating the index is given. In [30] Bezergianni and Georgakis introduce a modified version of the Harris index in which the closed loop performance is compared with that obtained with the best theoretical control action (minimum variance control) and no control action. In [4] Vishnubhotla et al. discuss a method of performance assessment based on the Harris index. The resulting index, gives an indication of the level of performance of the controller, and an indication of the action required to improve performance. In [24] Spring discusses a performance index based on minimum variance control. In [31] Ko and Edgar outline a scheme for the estimation of achievable PI control performance, measured by output variance, in linear processes with dead time when stochastic load disturbances are affecting the process.

A number of papers have been written in which modifications to the MVC benchmark have been made. In [32] Eriksson and Isaksson discuss how this technique provides an inadequate measure of performance if the aim is not control of statistically random disturbances. Some modifications to the
Harris index are suggested. In [33] Horch and Isaksson discuss a modification to the index introduced by Harris [23]. The modified index and the original index are then evaluated and compared using data from industrial processes. In [34] Isaksson discusses the MVC benchmarking technique and suggests a set of alternative indices. In [35] and [36] Huang discusses some of the aspects associated with the minimum variance control law for linear time variant processes. Alternative benchmarks that are more suitable for time variant processes are suggested. In [37] Venkatesan introduces a minimum variance feedback control algorithm (MVFCA) that can be used to calculate a series of adjustments required at the input that minimises the variance of the output variable. In [38] Kucera presents a tutorial paper emphasising the contribution of V. Peterka to the steady state minimum variance control problem. In [39] Qin presents an overview of the current status of control performance monitoring using minimum variance principles.

\section{Statistical ANALYSIS}

According to [1], the goal of statistical process monitoring (SPM) is to detect the existence, magnitude and time of occurrence of changes that cause a process to deviate from its desired operation. A number of useful techniques for the monitoring of process variables are discussed in this paper. These methods include Shewhart control charts, moving average control charts, cumulative sum charts and partial least squares methods.

The likelihood method is a useful technique for assessing performance. According to [1], this method may be used to determine if the error response characteristics are acceptable based on specified dynamic performance bounds. Dynamic response characteristics such as overshoot or settling time can be extracted from the pulse response of a fitted time series model of the output error. The pulse response of the estimated output error can be compared to the pulse response of the desired response specification to determine if the output error characteristics are acceptable. In [40] Tyler and Morari propose a framework in which acceptable performance is expressed by constraints on the closed loop transfer function impulse response coefficients. Using likelihood methods, a hypothesis test is outlined to determine if control deterioration has occurred. In [41] Zhang and Ho propose the use of the likelihood ratio method as a means of sensitivity analysis of stochastic system performance.

In [42] $\mathrm{Li}$ et al. develop a monitor to automatically detect poor control performance. The monitor provides a measure (Relative Performance Index - RPI) of a control loop performance relative to a reference model of acceptable control. The reference model simulates the controlled variable output of a user defined, acceptably tuned, control loop. In [43] Zhong demonstrates how to improve the effectiveness of equipment monitoring and process induced defect control through properly selecting, validating and using the hypothetical distribution models. In [44] Mosca and Agnoloni study the early detection problem of stability losses or close-toinstability conditions in feedback control systems, where the plant dynamics are uncertain and possibly time-varying. 


\section{OTHER ASSESSMENT TECHNIQUES}

This section contains a number of more 'problem specific' assessment techniques, as opposed to the more general methods discussed in previous sections. The focus of this section is on methods to both detect and diagnose oscillations in control loops. The techniques discussed here may well be considered special cases of the methods discussed in previous sections.

The first step in dealing with an under performing control loop with suspected oscillation disturbances is the detection stage. In [45] Hagglund presents a closed loop performance monitor (CLPM) to detect oscillations in the control loop. The procedure presented is automatic in the sense that no additional parameters, other than the normal controller parameters, have to be specified. In [46] Huang et al. discuss a method of determining the presence of oscillations in selected frequency ranges, based on the regularity of the zero crossings of filtered auto-covariance functions. In [47] Chang et al. present a system-wide dynamic performance monitoring system (DPMS), which includes special features such as oscillation detection. In [48] Stenman et al. propose a model-based method for detecting static friction (stiction) in control valves. In contrast to existing methods, only limited process knowledge is needed and it is not required that the loop has oscillating behaviour. In [49] Wallen proposes an integrated system for valve diagnostics and automatic PID tuning. The purpose of the method is to detect non-linearities such as friction and hysteresis since these may drastically decrease the control performance.

Once an oscillation has been detected, the next step is to determine its cause. In [50] Thornhill and Hagglund present a set of 'operational signatures' that indicate the cause of an oscillation. This method involves the offline analysis of ensembles of data from control loops. In [51] Horch proposes a simple method for the diagnosis of oscillations in process control loops based on the cross correlation between control variable and loop output. This method is shown to correctly identify the two most important reasons for oscillations in control loops in the process industry, namely, external oscillating disturbances and stiction in control valves. In [52] Taha et al. present an on line automatic procedure for the diagnosis of oscillations in control loops. This method works without disturbing normal plant operation.

\section{CONCLUSIONS}

According to [53], minimum variance control (MVC) as a benchmark (as discussed in [22]) or variants of it, is used in virtually all industrial controller assessment packages due to its theoretical and practical advantages. In [53] Hugo lists some of these software packages as follows: Performance assessment tool-kit [54]; loop scout [55]; Process Doc [56]; and Aspen Watch [57]. Software packages such as Probe [58] and Plant Triage [59] also offer a number of useful routines and algorithms related to MVC and some of the other assessment techniques mentioned previously. In [26] Harris et al. state that a comprehensive approach for assessing the effectiveness of control systems requires determination of the capability of the control system, development of suitable statistics for monitoring the performance of the existing system, development of methods for diagnosing the underlying causes for changes in the performance of the control system, and incorporation of these methods in an industrial setting.

The main advantage that MVC as a benchmark has over the other four categories discussed in the paper is that it not only gives an indication as to the current level of performance of the controlled system under investigation, but it can also determine whether or not current performance can be improved by retuning the controller. In [4] Vishnubhotla et al. highlight this point by stating that 'as a benchmark (MVC) ... provides useful information such as how well the current controller was tuned compared to the minimum variance controller and how much 'potential' there is to improve controller performance'. For example, an index (ratio of minimum achievable output variance to actual variance) value of 1 indicates that current performance cannot be improved by retuning the existing controller. However, an index value below 1 indicates retuning the controller will have an impact on improving system performance.

While time domain, frequency domain or statistical analysis techniques may give an accurate indication as to the current level of performance of the controller, no indication is given as to whether or not retuning will lead to improved performance. Simulations must be run and re-run with differently tuned parameters in order to determine if improved control is possible. This could prove to be an inefficient use of time if it was discovered, after numerous simulations had been run and analysed, that it is not possible to improve on the current control performance using the current controller structure.

Therefore, these findings would suggest that whatever assessment techniques are used, benchmarks specific to the controller under assessment must be used in order to determine whether retuning or controller redesign is necessary. According to [10], continuous performance monitoring requires benchmarking so that it may be observed how performance has changed with time. Also, this benchmark must be specific to the plant under investigation. Future work will focus on the development of a method to calculate controller specific benchmarks, in one of the assessment categories outlined in this paper, in order to provide a more efficient monitoring and assessment tool.

\section{REFERENCES}

[1] Cinar, A., and Undey, C. (1999). 'Statistical process and controller performance monitoring: A tutorial on current methods and future directions'. Proceedings of the American Control Conference, San Diego, California, pp. 2625-2639.

[2] Ender D. (1993). 'Process control performance: Not as good as you think'. Control Engineering, 40, (10), pp. 180-190.

[3] Jamsa-Jounela, S-L, Poikonen, R., Georgiev, Z., Zuehlke, U., Halmevaara, K. (2002). 'Evaluation of control performance: Methods and Applications' Proceedings of the 2002 IEEE International Conference on Control applications, Glasgow, Scotland. pp. 681-686.

[4] Vishnubhotla, A., Huang, B., and Shah, S.L., Badmus, L., (1999). 'Control loop performance assessment: An enterprise asset management solution'. Proceedings of International Conference and Exposition for Advancing Measurement, Triangle Park, NC, USA, pp. 31-40.

[5] Ju, Jun and Chiu, Min-Sen. (1998). 'A Fast Fourier Transform Approach for On-line Monitoring of the maximum 
Closed-Loop Log Modulus' Industrial and Engineering Chemistry Research, 37, pp. 1045-1050.

[6] Thyagarajan, T., Yu, C-C, Huang, H-P. (2003) 'Assessment of controller performance: a relay feedback approach'. Chemical Engineering Science, 58, pp. 497-512.

[7] Marlin, T.E. (2000). 'Process control: designing processes and control systems for dynamic performance'. McGraw - Hill; $2^{\text {nd }}$ Edition, 2000

[8] Swanda, A.P., and Seborg, D.E. (1999). 'Controller performance assessment based on setpoint response data'. Proceedings of the American Control Conference, Piscataway, NJ, USA, pp. 3863-7.

[9] Horch A, Stattin A. (2002). A complete practical implementation of a method for step response performance assessment. Proceedings of the IEE Seminar Control Loop Performance Assessment. London, UK, pp. 4/1-5.

[10] Ruel, M. (2002). 'Learn How To Assess And Improve Control Loop Performance', ISA, Chicago.TOP Control www.topcontrol.com.

[11] Huang, H.P., and Jeng, J.C. (2002). 'Monitoring and assessment of control performance for single loop systems'. Industrial and Engineering Chemistry Research, 41, pp. 12971309

[12] Stanfelji, N., Marlin T.E., MacGregor, J.F. (1993). 'Monitoring and diagnosing process control performance: The single loop case'. Industrial Engineering Chemistry Research, 1993, (32), pp. 301-314.

[13] Hagglund, T., (1999). 'Automatic detection of sluggish control loops'. Control Engineering Practice, 7, pp. 15051511.

[14] Belanger, P.W. and Luyben, W.L. (1996) 'A new test for the evaluation of the regulatory performance of controlled processes'. Industrial and Engineering Chemistry Research, 35, pp. 3447-3457.

[15] Luyben, W.L. and Luyben, M.L. (1996). 'Essentials of process control'. McGraw-Hill Science/Engineering/Math

[16] Astrom, K.J., Hagglund, T. (1984) 'Automatic tuning of simple regulators with specifications on phase and amplitude margins'. Automatica, 20 (5), pp. 645-651.

[17] Chiou, R-C. and Yu, C-C. (1993) 'Monitoring procedure for intelligent control: on-line identification of maximum closed loop log modulus'. Industrial and Engineering Chemistry Research, 32, pp. 90-99.

[18] Elliot, T.R., and Luyben, W.L. (1995). 'Capacity-based economic-approach for the quantitative assessment of process controllability during the conceptual design stage'. Industrial and Engineering Chemistry Research, 34 (11), pp. 3907 3915.

[19] Elliott TR, Luyben WL, Luyben ML. (1997). 'Application of the capacity-based economic approach to an industrialscale process'. Industrial and Engineering Chemistry Research, 36 (5), pp. 1727-1737.

[20] Elliott TR, Luyben WL. (1996). 'Quantitative assessment of controllability during the design of a ternary system with two recycle streams'. Industrial and Engineering Chemistry Research, 35 (10), pp. 3470-3479.

[21] Kendra, S.J., and Cinar, A. (1997). 'Controller performance assessment by frequency domain techniques'. Journal of Process Control, 7(3), pp. 181-194.

[22] Desborough, L. and Harris, T.J. (1992). 'Performance assessment measures for univariate feedback control'. Canadian Journal of Chemical Engineering, 70, pp. 11861197.

[23] Harris, T.J. (1989). 'Assessment of control loop performance'. Canadian Journal of Chemical Engineering, 67, pp. 856-861.

[24] Spring R. (1996). 'Minimum variance as a benchmark for feedback control'. Tappi Journal 79 (8), pp. 97-101.

[25] Thornhill, N.F., Shah, S.L., and Huang, B. (2000) 'Controller performance assessment in setpoint tracking and regulatory control'. International Journal of Adaptive Control and Signal Processing, 17, pp. 709-727.

[26] Harris, T.J., Seppala, C.T., and Desborough, L.D., (1999) 'A review of performance monitoring and assessment techniques for univariate and multivariate control systems'. Journal of Process Control, 9 (1), pp. 1-17.

[27] Grimble, M.J. (2002). 'Controller performance benchmarking and tuning using generalised minimum variance control'. Automatica, 38, pp. 2111-2119.

[28] Huang, B. and Shah, S.L. (1999). 'Performance assessment of control loops: Theory and applications'. Springer-Verlag; ISBN: 1-85233-639-0.

[29] Desborough, L. and Harris, T.J, (1993). 'Performance assessment measures for univariate feedforward/feedback control'. Canadian Journal of Chemical Engineering, 71, pp. 605-616.
[30] Bezergianni S, Georgakis C. (2000). 'Controller performance assessment based on minimum and open-loop output variance'. Control Engineering Practice, 8 (7), pp. 791-797.

[31] Ko, B-S. and Edgar, T.F. (1998). 'Assessment of achievable PI control performance for linear processes with dead time'. Proceedings of the American Control Conference, Evanston, IL, USA, pp. 1548-52.

[32] Eriksson, P-G., and Isaksson, A.J., (1994). 'Some aspects of control loop performance monitoring'. Proceedings of the $3^{\text {rd }}$ IEEE Conference on Control Applications, Glasgow, Scotland, pp. 1029-1034.

[33] Horch, A., and Isaksson, A.J. (1998). 'A modified index for control performance assessment'. Proceedings of the American Control Conference, Philadelphia, Pennsylvania, pp. 475-83.

[34] Isaksson AJ. (1996). 'PID controller performance assessment'. Proceedings of Control Systems '96. (Preprints), Montreal, Que., Canada, pp.163-70.

[35] Huang B. (1999). 'Performance assessment of processes with abrupt changes of disturbances'. Canadian Journal of Chemical Engineering, 77 (5), pp. 1044-1054.

[36] Huang B. (2002). 'Minimum variance control and performance assessment of time-variant processes'. Journal of Process Control, 12 (6), pp. 707-719.

[37] Venkatesan G. (2002). 'An algorithm for minimum variance control of a dynamic time-delay system to reduce product variability'. Computers and Electrical Engineering, 28 (3), pp. 229-239.

[38] Kucera V. (1999). 'Minimum variance control: A homage to Peterka'.International Journal of Adaptive Control, 13 (6), pp. 433-449.

[39] Qin, S.J. (1998). ' Control performance monitoring - a review and assessment'. Computers and Chemical Engineering, 23, pp.173-186.

[40] Tyler, M.L., and Morari, M., (1995). 'Performance Monitoring of Control Systems using Likelihood Methods' Proceedings of the American Control Conference, Evanston, IL, USA, pp. 1245-9.

[41] Zhang, B. and Ho, Y-C. (1989). 'Variance reduction for likelihood-ratio method'. Proceedings of the 28th IEEE Conference on Decision and Control, 1, pp. $145-150$.

[42] Li Q, Whiteley JR, Rhinehart RR . (2003). 'A relative performance monitor for process controllers'. International Journal of Adaptive Control, 17 (7-9), pp. 685-708.

[43] Zhong, Lei. (2003). 'Defect distribution model validation and effective process control'.Proceedings of Spie - the International Society for Optical Engineering, USA, pp.31-8.

[44] Mosca, E., and Agnoloni, T. (2003). 'Closed loop monitoring for early detection of performance losses in feedback control systems'. Automatica, 39, pp.2071-2084.

[45] Hagglund, T. (1995). 'A control loop performance monitor', Control Engineering Practice, 3, pp. 1543-1551.

[46] Huang, B., Thornhill, N.F., and Zhang, H., (2001). 'Detection of multiple oscillations in control loops'. Journal of Process Control, accepted for publication.

[47] Chang, C.L., Liu, A.S., Chen, Y.T. (1993). 'Dynamic performance monitoring-system of taiwan-power-company' IEEE Transactions on Power Systems, 8 (3), pp. 815-822.

[48] Stenman A, Gustafsson F, Forsman K. (2003). 'A segmentation-based method for detection of stiction in control valves'. International Journal of Adaptive Control, 17 (7-9), pp. 625-634

[49] Wallen, A., (1997). 'Valve diagnostics and automatic tuning'. Proceedings of the American Control Conference, Albuquerque, New Mexico, pp. 2930-2934.

[50] Thornhill, N.F., and Hagglund, T. (1997). 'Detection and diagnosis of oscillation in control loops'. Control Engineering Practice, 5, pp. 1343-1354.

[51] Horch, A., (1999). 'A simple method for oscillation diagnosis in process control loops'. Proceedings of the 1999 IEEE International Conference on Control Applications, Piscataway, NJ, USA, pp. 807-12.

[52] Taha, O., Dumont, G.A., and Davies, M.S., (1996). 'Detection and diagnosis of oscillations in control loops'. Proceedings of the $35^{\text {th }}$ IEEE Conference on Decision and Control, Kobe, Japan, pp. 2432-2437.

[53] Hugo, A.J., (2001) 'Process controller performance monitoring and assessment'. Hydrocarbon processing, April. Control Arts website, www.controlartsinc.com.

[54] Control Arts Inc. (2000), Performance assessment tool-kit, www.controlarstinc.com.

[55] Honeywell (2000), Loop Scout, www.IAC.Honeywell.com

[56] Matrikon, (2000) Process Doc, www.Matrikon.com.

[57] Aspen watch, (2000). www.aspentech.com.

[58] ACTC, Advanced Control Technology Club, www.iscltd.com/actclub.

[59] ExperTune. www.expertune.com 